\title{
Leukotriene D4 nasal provocation test: Rationale, methodology and diagnostic value
}

\author{
ZHENG ZHU ${ }^{1}$, YANQING XIE ${ }^{1}$, WEIJIE GUAN ${ }^{1}$, YI GAO ${ }^{1}$, SHU XIA $^{1}$, JIANXIN LIANG $^{2}$ and JINPING ZHENG ${ }^{1}$ \\ ${ }^{1}$ State Key Laboratory of Respiratory Disease, China Clinical Research Center of Respiratory Disease; \\ ${ }^{2}$ Department of Otorhinolaryngology, First Affiliated Hospital, Guangzhou Medical University, \\ Guangzhou, Guangdong 510120, P.R. China
}

Received September 27, 2014; Accepted April 5, 2016

DOI: $10.3892 /$ etm.2016.3324

\begin{abstract}
Cysteinyl leukotrienes (LT) play a vital role in the pathogenesis of allergic rhinitis (AR), but few studies have investigated the nasal mucosal physiological response to LTs in AR patients. The aim of the present study was to establish the methodology and investigate the diagnostic value and safety of a leukotriene D4 (LTD4) nasal provocation test. LTD4 nasal provocation tests were performed in $26 \mathrm{AR}$ patients and 16 normal control subjects. Nasal airway responsiveness was assessed by calculating the concentration of LTD4 required to cause a $60 \%$ increase in nasal airway resistance ( $\left.\mathrm{PC}_{60} \mathrm{NAR}-\mathrm{LTD} 4\right)$, which was measured using rhinomanometry and a composite symptom score. Receiver operating characteristic (ROC) curve was applied to evaluate the diagnostic value of LTD4 nasal provocation test, and adverse events were recorded. The study protocol was registered with the U.S. National Institutes of Health (https://clinicaltrials. gov/ct2/show/NCT01963741). PC ${ }_{60}$ NAR-LTD4 in AR was significantly lower compared with that of normal controls [8.36 (IQR, 10.00) vs. $17.00(\mathrm{IQR}, 0.00) \mu \mathrm{g} / \mathrm{ml}, \mathrm{P}=0.005]$. Composite symptom score was higher in AR as compared with normal controls $(1.19 \pm 0.94$ vs. $0.12 \pm 0.50, \mathrm{P}<0.001)$. The symptom scores included sneezing $(0.12 \pm 0.34$ vs. $0.00 \pm 0.00$, $\mathrm{P}=0.149)$, rhinorrhea $(0.79 \pm 0.66$ vs. $0.06 \pm 0.25, \mathrm{P}<0.001)$ and chemosis or itching of the eyes $(0.06 \pm 0.25$ vs. $0.21 \pm 0.42$, $\mathrm{P}=0.216)$. High diagnostic value was indicated by the ROC [AUC: 0.822 , 95\% CI $(0.665,0.961)]$. No serious adverse events were observed. Thus, the present results indicate that AR patients exhibited nasal hyperactivity to LTD4, and the established procedure of LTD4 nasal provocation testing is effective and safe for use in the diagnosis of AR.
\end{abstract}

Correspondence to: Professor Jinping Zheng, State Key Laboratory of Respiratory Disease, China Clinical Research Center of Respiratory Disease, First Affiliated Hospital, Guangzhou Medical University, 151 Yanjiang Road, Guangzhou, Guangdong 510120, P.R. China

E-mail: jpzhenggy@163.com

Key words: allergic rhinitis, leukotriene D4, provocation test, hyperresponsiveness, methodology

\section{Introduction}

The levels of cysteinyl leukotrienes(LTs) in nasal secretions are elevated following allergen challenge or during natural exposure to allergens in allergic rhinitis (AR) patients $(1,2)$. LTs play a vital role in the pathogenesis of AR and asthma $(3,4)$, and as asthma patients frequently exhibit AR, patients that suffered from asthma and AR were particular good candidates for anti-LT therapy (5).

Anti-LT therapy has been indicated to be effective in relieving the symptoms of AR or asthma in large-scale randomized, double-blind, placebo-controlled clinical trials $(6,7)$. Nasal congestion and rhinorrhea in AR were reduced via the administration of montelukast, a LT receptor antagonist (LTRA), to a degree similar to that of antihistamines $(8,9)$; however, the results of other trials were inconsistent with this $(6,7,10)$. It remains unclear which population group may benefit most since the efficacy of the LTRA varied among asthmatic patients. Since it is difficult to predict responsiveness to anti-LT therapy in an individual patient, it is critical to develop a simplified method to identify the treatment response.

LTC and LTD can induce an increase in nasal mucosal blood flow and nasal airway resistance (NAR); however, inhalation of LTD4 is associated with higher potency (11) and slow deactivation in vivo (12) as compared with LTC4 and LTE4. It has been reported that LTC4 is $~ 10$ times (13), while LTD4 was 5,000 times, as potent as histamine in achieving a $150 \%$ increase in NAR (14), as measured by rhinomanometry. Furthermore, LTD4 has been suggested to be an effective bronchial provocation agent in our previous studies $(15,16)$. Consequently, LTD4 is speculated to be a potentially useful provocation agent (15). To the best of our knowledge, few prior studies have investigated the nasal physiological response to LTs.

The present pilot study aimed to establish the procedure for an LTD4 nasal provocation test (LTD4-NAPT), and to investigate the clinical characteristics, diagnostic value and safety profiles of nasal response to LTD4.

\section{Patients and methods}

Subjects. Between November 2012 and May 2013, patients aged 18-30 years with recurrent symptoms of sneezing, rhinorrhea, 
nasal stuffiness or itching during the preceding years were recruited from the First Affiliated Hospital of Guangzhou Medical University (Guangzhou, China). AR patients were positive to at least one kind of inhaled allergens by skin prick test (ALK-Abelló, Hørsholm, Denmark). The diagnosis of AR was based on ARIA 2008 (17). Exclusion criteria included: Smoking status; NAR increased $>30 \%$ after ethanol diluent challenge; a past confirmed history of chronic respiratory disease other than asthma; undergoing immunotherapy; inability to complete the test or limited understanding of the test; acute upper and lower airway infections two weeks prior to the enrollment; and other severe systemic diseases, such as myocardial infarction or malignant tumor. The patients were required to abstain from the use of oral or nasal anti-histamines and LTRA for at least one week, and oral, nasal and inhaled corticosteroids for at least two weeks, since these drugs may affect the study results. The normal control subjects were aged between 18 and 30 years, had no upper and lower respiratory tract infections for the previous two weeks, and no allergic or systemic diseases.

The study protocol was approved by the Ethics Committee of the First Affiliated Hospital of Guangzhou Medical University. The purpose, test procedures, potential benefits and safety were explained to the participants and all subjects signed written informed consent prior to the study.

Study design. This was an open-labeled study. Nasal airway responsiveness was assessed by the increase of NAR and a composite symptom score, and positive response was defined as the provocative concentration of LTD4 causing a $60 \%$ increase in total NAR ( $\left.\mathrm{PC}_{60} \mathrm{NAR}-\mathrm{LTD} 4\right)$ no more than $3.2 \times 10^{4} \mathrm{nmol} / 1$, or the symptom score of $>3$ points (pts) during the test (18).

Rhinomanometry. Total nasal airway inspiration resistance (NAR), left and right nasal airway inspiration resistance (RIL and RIR) were measured by rhinomanometry (JAEGER MasterScope; CareFusion Co., Ltd., Hoechberg, Germany). A total of at least three repeatability (no more than five) rhinomanometry maneuvers were performed in each nostril, with each maneuver contains at least three respiratory cycles to calculate the mean value of NAR, under a pressure of $150 \mathrm{~Pa}$.

Preparation of diluents. The preparation of LTD4 simulative diluents was almost the same as described elsewhere $(15,16)$. LTD4 (100 $\mu \mathrm{g} / \mathrm{ml}$ in ethanol; Cayman Chemical Company, Ann Arbor, MI, USA) was stored at $-80^{\circ} \mathrm{C}$. The diluent of $16 \mu \mathrm{g} / \mathrm{ml}$ LTD4 (solution A) was prepared by adding $0.504 \mathrm{ml}$ normal saline into $0.096 \mathrm{ml}$ LTD4 aliquot. An aliquot of solution A was added to $0.3 \mathrm{ml}$ normal saline, forming $8 \mu \mathrm{g} / \mathrm{ml}$ LTD4 diluent (solution B). An aliquot of solution B was added into $0.2 \mathrm{ml}$ normal saline forming $4 \mu \mathrm{g} / \mathrm{ml}$ LTD4 diluent (solution C). Ethanol diluent control was also prepared except that $0.096 \mathrm{ml}$ ethanol absolute was adopted instead of LTD4. To ensure the quality of the test, LTD4 diluents should be discarded at the end of day, as stated in the manufacturers' specifications (Cayman Chemical Company).

Nasal challenge. Diluents were delivered via nasal spray pumps (Dezong Pharmaceutical, Co., Ltd., Foshan, China) by compressed air $(0.1 \mathrm{ml}$ per spray), with provocative concentration increased in a step-wise manner. Nasal challenge using $16 \%$ ethanol diluent, the concentration of which corresponded to solution A, was performed in order to exclude subjects hypersensitive to ethanol or saline. The LTD4 challenge could be initiated provided that NAR increase was $<30 \%$. A range between 4 and $16 \mu \mathrm{g} / \mathrm{ml}$ LTD4 diluents were applied for a double-fold increment approach at intervals of $6 \mathrm{~min}$. These procedures were terminated in the case of $a \geq 60 \%$ NAR increase or a composite symptom score $>3$, or until the use of the last concentration of LTD4 diluents.

Symptom score. A composite symptom score according to Riechelmann et al (18) was applied: 3-5 Sneezes $=1 \mathrm{pt}$; $>5$ sneezes $=2$ pts; rhinorrhea $<1 \mathrm{ml}=1 \mathrm{pt}$; rhinorrhea $>1 \mathrm{ml}=2$ pts; pruritus of the palate, ears or eyes $=1 \mathrm{pt}$; and conjunctivitis, cough, urticaria or difficult breathing $=2$ pts. Total scores ranged between 0 and 6 pts. The provocation test was positive when the total score reached $>3$ pts, whatever the changes of NAR.

Determination of positive response. Positive response of LTD4 nasal provocation test was defined as an increase in NAR of $\geq 60 \%$ by the concentration of LTD 4 no more than $3.2 \times 10^{4} \mathrm{nmol} / 1$ and/or composite symptom score $>3$ pts.

Statistical analysis. Statistical analysis was performed using SPSS software, version 16.0 (SPSS, Inc., Chicago, IL, USA). Dot plots were produced using GraphPad Prism, version 5.01 (GraphPad Software, Inc., San Diego, CA, USA). Mean \pm standard deviation $(x \pm s)$ was adopted for data with normal distribution, otherwise median (interquartile range) was used. Two-sided $t$-tests were performed for comparison of baseline levels. A dot plot was applied for distribution character of $\mathrm{PC}_{60}$ NAR-LTD4. A row-column table was analyzed via $\chi^{2}$ test. The diagnostic value was assessed using receiver operating characteristic curve (ROC), with area under curve (AUC) and $95 \%$ confidence interval (CI) being reported. Diagnostic value and adverse events were analyzed for efficacy and safety outcomes. Statistical significance was defined as $\mathrm{P}<0.05$.

\section{Results}

Demographics and changes in RIR, RIL and NAR. Among the 50 subjects that underwent screening, 8 subjects were withdrawn for being unwilling to undergo a nasal provocation. A total of 26 AR patients and 16 normal control subjects were enrolled. Baseline demographics were comparable between AR and normal controls (all P>0.05, Table I).

Prior to challenge, NAR was slightly higher in the AR group compared with the controls, but without statistically significant differences (Table I). Following challenge, marked increases in RIL, RIR and NAR in both groups were observed, significantly higher in AR patients compared with the normal controls (all $\mathrm{P}<0.05$, Fig. 1).

Changes in $P_{60} N A R-L T D 4$ in response to LTD4 challenge. Positive responses to LTD4 challenge were observed in 19 AR patients and 2 normal controls (positive rate, 73.08 vs. $12.50 \%$, $\mathrm{P}<0.001)$. AR had a markedly lower median of $\mathrm{PC}_{60} \mathrm{NAR}-\mathrm{LTD} 4$ $(8.36 \mu \mathrm{g} / \mathrm{ml})$, with a larger interquartile range $(10.00 \mu \mathrm{g} / \mathrm{ml})$ 
Table I. Baseline demographic characteristics and nasal airway resistance in allergic rhinitis and normal controls.

\begin{tabular}{lccc}
\hline Parameter & Normal control & AR & P-value \\
\hline Patients & 16 & 26 & - \\
Age (years) & $21.1 \pm 3.2$ & $22.2 \pm 6.5$ & 0.440 \\
Male/female & $11 / 5$ & $9 / 17$ & 0.055 \\
Height $(\mathrm{cm})$ & $165.8 \pm 8.5$ & $163.9 \pm 8.5$ & 0.493 \\
Weight $(\mathrm{kg})$ & $56.5 \pm 7.4$ & $53.15 \pm 7.1$ & 0.151 \\
RIL & $0.41 \pm 0.17$ & $0.56 \pm 0.34$ & 0.123 \\
RIR & $0.47 \pm 0.40$ & $0.63 \pm 0.22$ & 0.097 \\
NAR & $0.22 \pm 0.08$ & $0.28 \pm 0.32$ & 0.061 \\
\hline
\end{tabular}

AR, allergic rhinitis; RIL, nasal airway inspiration resistance at left side; RIR, nasal airway inspiration resistance right side; NAR, total nasal airway inspiration resistance.
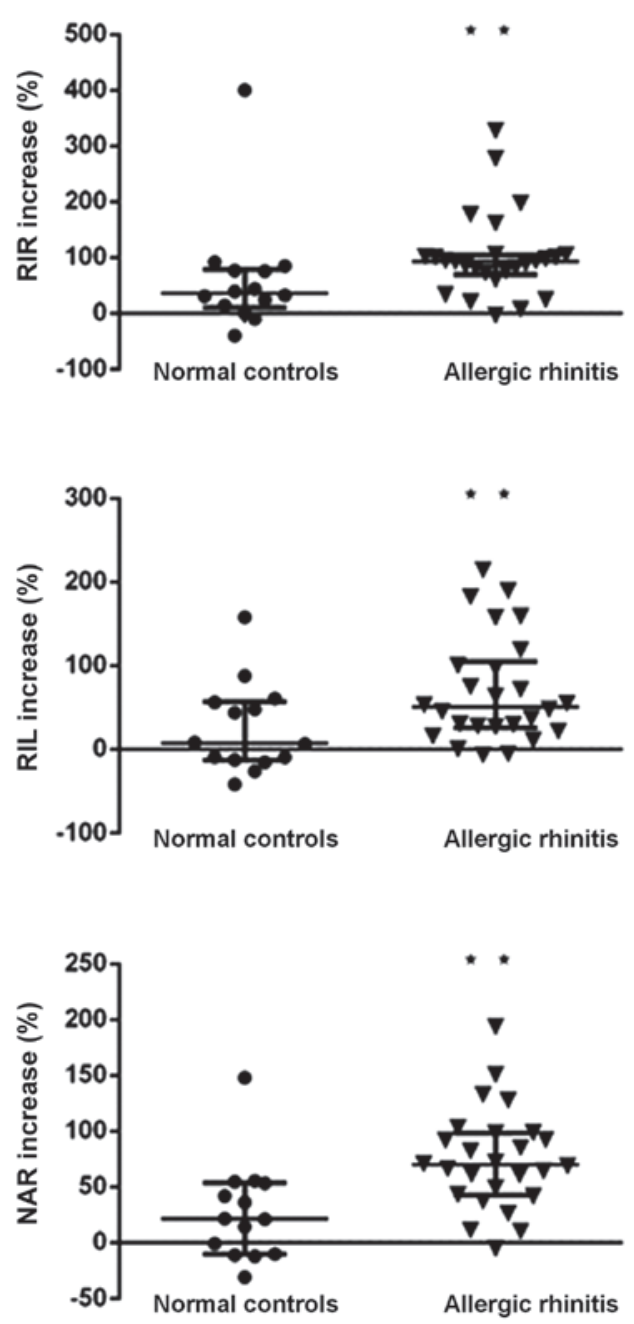

Figure 1. Individual increase (\%) of nasal airway resistance after nasal challenge in allergic rhinitis patients and normal controls. ${ }^{* *} \mathrm{P}<0.05$. RIR, nasal airway inspiration resistance right side; RIL, nasal airway inspiration resistance at left side; NAR, total nasal airway inspiration resistance.

as compared with normal controls (Fig. 2). PC ${ }_{60}$ NAR-LTD4 varied between 1.17 and $11.75 \mu \mathrm{g} / \mathrm{ml}$ in $19 \mathrm{AR}$, by comparison, $\mathrm{PC}_{60} \mathrm{NAR}-L T D 4$ was $>16.00 \mu \mathrm{g} / \mathrm{ml}$ in all normal controls, with

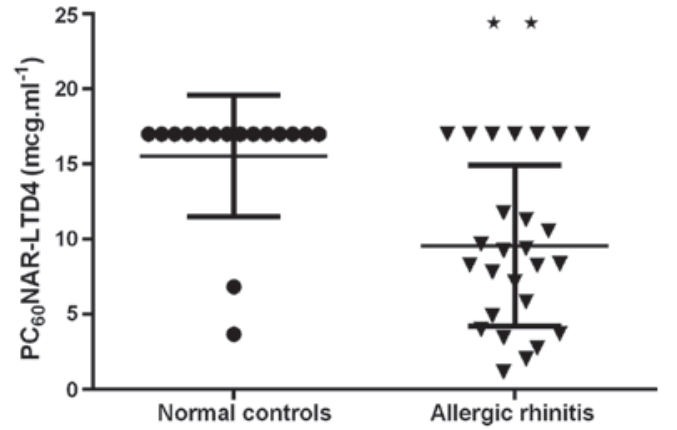

Figure 2. $\mathrm{PC}_{60} \mathrm{NAR}-\mathrm{LTD} 4$ in allergic rhinitis and normal controls. ${ }^{* *} \mathrm{P}<0.05$. $\mathrm{PC}_{60}$ NAR-LTD4, concentration of LTD4 required to cause a $60 \%$ increase in nasal airway resistance.

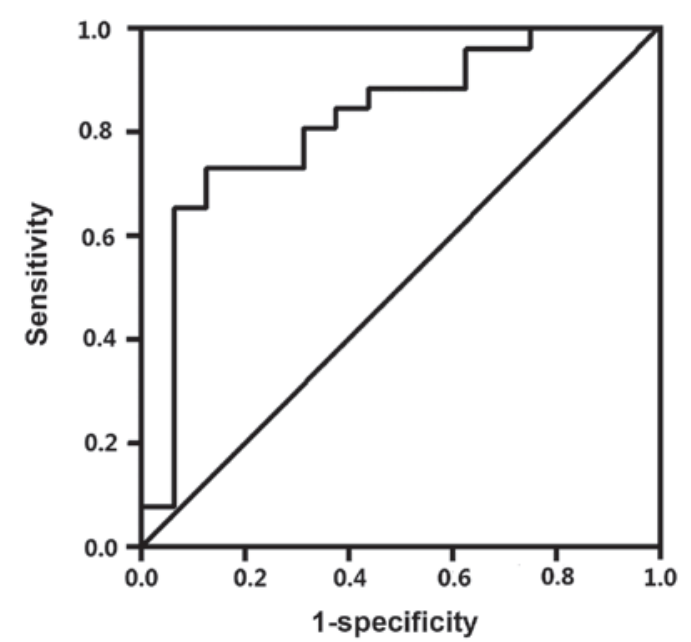

Figure 3. Receiver operating characteristic curve of leukotriene D4 nasal provocation test assessed based on the increase of nasal airway resistance.

two exceptions of 3.68 and $6.84 \mu \mathrm{g} / \mathrm{ml}$. The increase of NAR was negatively correlated with $\log _{10}\left(\mathrm{PC}_{60} \mathrm{NAR}-\mathrm{LTD} 4\right)$ in $\mathrm{AR}$ $(\mathrm{r}=-0.75, \mathrm{P}<0.001)$. Mean NAR increase was $74.44 \pm 44.95 \%$ (range, 61.38-193.68\%) in AR patients that were positive to LTD4 challenge.

Changes in symptom scores in response to LTD4 challenge. The symptom scores were significantly higher in AR group compared with in the control group $(1.19 \pm 0.94$ vs. $0.12 \pm 0.50$, $\mathrm{P}<0.001)$. The symptom scores consisted of sneezing $(0.12 \pm 0.34$ vs. $0.00 \pm 0.00, P=0.149)$, rhinorrhea $(0.79 \pm 0.66$ vs. $0.06 \pm 0.25, \mathrm{P}<0.001)$ and chemosis or itching of the eyes $(0.06 \pm 0.25$ vs. $0.21 \pm 0.42, P=0.216)$. No symptom scores $>3$ pts were detected in either group. AUC was 0.822 [95\% CI, (0.665, 0.961)] (Fig. 3).

Adverse effects induced by LTD4 challenge. Adverse events were observed in 3 AR patients that were positive to LTD4 nasal provocation. The major adverse events by incidence were eye itching (3/3), tears (3/3) and full nasal congestion (1/3) with breathing through mouth. All adverse events were tolerated and recovered within an hour after challenge. No serious adverse events (including wheezing or induced bronchia constriction) were observed. 


\section{Discussion}

The present study demonstrated that LTD4 was able to induce an increase in NAR and nasal discharge in patients with AR compared with normal controls. The increase of NAR was negatively correlated with $\log _{10}\left(\mathrm{PC}_{60} \mathrm{NAR}\right.$-LTD4) $(\mathrm{r}=-0.75, \mathrm{P}<0.001)$. The majority of AR patients $(19 / 26)$ were nasal airway hyperactive to LTD4, high diagnostic value of LTD4-NPT was indicated by the ROC (AUC, 0.813). Bisgaard et al (13) demonstrated that, even in normal control subjects, a high dose of LTD4 may induce nasal mucosal blood flow and NAR in the absence of nasal discharge. Furthermore, Numata et al (19) reported that nasal mucosal swelling induced by nasal challenge was prevented by LTRA administration.

To the best of our knowledge, the present study was the first to directly and quantitatively evaluate the effects of LTD4 on NAR and nasal symptoms in patients with AR. The results supported our hypothesis that LT impacts the upper and lower airways, and that LTD4 may be employed as a nasal provocative mediator.

By comparison, LTD4 provocation test did not cause sneezing and itching or other irritative symptoms in the present study, which was similar to some previous studies $(20,21)$. Miadonna et al (20) and Howarth (21) demonstrated that the symptoms of AR such as nasal secretion, nasal obstruction in particular, was more strongly correlated with the release of arachidonic acid metabolites than histamine in the nasal secretion or specimen during natural exposure. However, when stimulated with histamine or cysLT there was a different result; sneezing, itching, rhinorrhea and nasal obstruction could be induced by histamine rapidly (within a few seconds), while cysLT caused a more pronounced and longer lasting nasal obstruction $(13,19)$. These phenomena implied that not only one, probably many mediators were involved in the development of symptoms, even involved with nervous reflex.

To date, a number of methods, such as the measurement of LTs in urinal, nasal lavage or blood, have been used to identify the patients with AR or asthma that are sensitive to LTRA $(22,23)$; however, none of them has been successfully used in clinical practice.

Although the cost of LTD4-NPT is higher than that of histamine/methacholine-NPT, LTD4-NPT may provide improved predictive efficacy of LTRA among AR patients with distinct inflammatory phenotypes compared with conventional provocation agents (such as histamine) or for identifying patients who are highly sensitivity to LTs. Furthermore, by measuring the alterations in upper and lower airway responsiveness and inflammatory mediators to LTD4-NPT, more may be elucidated regarding the association between AR and asthma. These critiques will be investigated in our future studies.

There are a number of limitations in the present study, owing to high thermosensitivity and reducibility in aqueous environment; the LTD4 diluents were valid for only one day, which may limit the widely use of it. Furthermore, it is necessary to increase the population size in future studies.

In conclusion, the established procedure of LTD4 nasal provocation test is effective in the evaluation of nasal airway hyperresponsiveness, and is well tolerated. LTD4-NAPT may be applied in the diagnosis of AR and provide a useful tool for testing the effectiveness of LTRA in future.

\section{Acknowledgements}

The authors would like to thank Dr Xu Shi and Mr. Wenhua Jian from the First Affiliated Hospital of Guangzhou Medical University; Mrs. Qingxia Liu from Qingyuan People's Hospital (Qingyuan, China); Miss E Guo from Xiangyang Central Hospital (Xiangyang, China); Miss Zhiyu Liang from Yuexiu People's Hospital (Guangzhou, China); and Miss Linting Luo, Mr. Diteng Luo, Mr. Xiangong Xu, Mr. Huayi Huang, Miss Yongqing Ye and Miss Xianmiao Ye from Guangzhou Medical University for their assistance with recruiting participants. This study was supported by Changjiang Scholars and Innovative Research Team in University (grant no. ITR0961), The National Key Technology R\&D Program of the 12th National Five-year Development Plan (grant no. 2012BAI05B00) and Guangzhou Medical University Doctor Startup Items (grant no. 2011C39).

\section{References}

1. Bisgaard H, Robinson C, Rømeling F, Mygind N, Church M and Holgate ST: Leukotriene $\mathrm{C} 4$ and histamine in early allergic reaction in the nose. Allergy 43: 219-227, 1988

2. Kunitomo M and Otsuka H: Comparison of antigen-induced leukotriene and histamine release from nasal scrapings in allergic rhinitis. Rhinology 43: 199-204, 2005.

3. Busse WW: The role of leukotrienes in asthma and allergic rhinitis. Clin Exp Allergy 26: 868-879, 1996.

4. Howarth PH, Salagean M and Dokic D: Allergic rhinitis: Not purely a histamine-related disease. Allergy 55 (Suppl 64): S7-S16, 2000.

5. Price DB, Swern A, Tozzi CA, Philip G and Polos P: Effect of montelukast on lung function in asthma patients with allergic rhinitis: Analysis from the COMPACT trial. Allergy 61: 737-742, 2006.

6. Patel P, Philip G, Yang W, Call R, Horak F, LaForce C, Gilles L, Garrett GC, Dass SB, Knorr BA and Reiss TF: Randomized, double-blind, placebo-controlled study of montelukast for treating perennial allergic rhinitis. Ann Allergy Asthma Immunol 95: 551-557, 2005.

7. Philip G, Williams-Herman D, Patel P, Weinstein SF, Alon A, Gilles L, Tozzi CA, Dass SB and Reiss TF: Efficacy of montelukast for treating perennial allergic rhinitis. Allergy Asthma Proc 28: 296-304, 2007.

8. Pinar E, Eryigit O, Oncel S, Calli C, Yilmaz O and Yuksel H: Efficacy of nasal corticosteroids alone or combined with antihistamines or montelukast in treatment of allergic rhinitis. Auris Nasus Larynx 35: 61-66, 2008.

9. Nayak A and Langdon RB: Montelukast in the treatment of allergic rhinitis: An evidence-based review. Drugs 67: 887-901, 2007.

10. Virchow JC and Bachert C: Efficacy and safety of montelukast in adults with asthma and allergic rhinitis. Respir Med 100: 1952-1959, 2006.

11. Drazen JM: Leukotrienes as mediators of airway obstruction. Am J Respir Crit Care Med 158: S193-S200, 1998.

12. Lee TH, Woszczek G and Farooque SP: Leukotriene E4: Perspective on the forgotten mediator. J Allergy Clin Immunol 124: 417-421, 2009.

13. Bisgaard H, Olsson P and Bende M: Effect of leukotriene D4 on nasal mucosal blood flow, nasal airway resistance and nasal secretion in humans. Clin Allergy 16: 289-297, 1986.

14. Miadonna A, Tedeschi A, Leggieri E, Lorini M, Folco G, Sala A, Qualizza R, Froldi M and Zanussi C: Behavior and clinical relevance of histamine and leukotrienes C4 and B4 in grass pollen-induced rhinitis. Am Rev Respir Dis 136: 357-362, 1987.

15. Guan WJ, Zheng JP, Gao Y, Jiang CY, An JY, Yu XX and Liu WT: Leukotriene D4 bronchial provocation test: Methodology and diagnostic value. Curr Med Res Opin 28: 797-803, 2012.

16. Guan WJ, Zheng JP, Gao Y, Jiang C, Xie Y, An J, Yu X, Liu W and Zhong N: Leukotriene D4 and methacholine bronchial provocation test for identifying leukotriene-responsiveness subtypes. J Allergy Clin Immunol 131: 332-338, 2013. 
17. Bousquet J, Khaltaev N, Cruz AA, Denburg J, Fokkens WJ, Togias A, Zuberbier T, Baena-Cagnani CE, Canonica GW, van Weel C, et al: Allergic rhinitis and its impact on asthma (ARIA) 2008 Update (in collaboration with the World Health Organization, GA(2)LEN and AllerGen). Allergy 63 (Suppl 86): 8-160, 2008.

18. Riechelmann H, Bachert C, Goldschmidt O, Hauswald B, Klimek L, Schlenter WW, Tasman AJ and Wagenmann M German Society for Allergology and Clinical Immunology (ENT Section); Working Team for Clinical Immunology: Application of the nasal provocation test on diseases of the upper airways. Position paper of the German society for allergology and clinical immunology (ENT Section) in cooperation with the working Team for Clinical Immunology. Laryngorhinootologie 82: 183-188, 2003 (In German).

19. Numata T, Hanazawa T, Konno A, Terada N, Yamakoshi T and Nagata H: Comparative role of peptide leukotrienes and histamine in the development of nasal mucosal swelling in nasal allergy. Ann Otol Rhinol Laryngol 108: 467-473, 1999.
20. Miadonna A, Tedeschi A, Leggieri E, Lorini M, Folco G, Sala A, Qualizza R, Froldi M and Zanussi C: Behavior and clinical relevance of histamine and leukotrienes C4 and B4 in grass pollen-induced rhinitis. Am Rev Respir Dis 136: 357-362, 1987.

21. Howarth PH: Mediators of nasal blockage in allergic rhinitis. Allergy 52 (Suppl 40): S12-S18, 1997.

22. Serrano CD, Valero A, Bartra J, Roca-Ferrer J, Muñoz-Cano R, Sánchez-López J, Mullol J and Picado C: Nasal and bronchial inflammation after nasal allergen challenge: Assessment using noninvasive methods. J Investig Allergol Clin Immunol 22: 351-356, 2012.

23. Baek HS, Cho J, Kim JH, Oh JW and Lee HB: Ratio of leukotriene e(4) to exhaled nitric oxide and the therapeutic response in children with exercise-induced bronchoconstriction. Allergy Asthma Immunol Res 5: 26-33, 2013. 